\title{
Identification of novel species-specific antigens of Mycoplasma hyopneumoniae by preparative SDS-PAGE ELISA profiling
}

\author{
Anthony L. Scarman, ${ }^{1,2}$ James C. Chin, ${ }^{1}$ Graeme J. Eamens, ${ }^{1}$ \\ Stephen F. Delaney ${ }^{2}$ and Steven P. Djordjevic ${ }^{1}$
}

Author for correspondence: Steven P. Djordjevic. Tel: +61 46 293426/349. Fax: +61 46293384. e-mail: djordjs@agric.nsw.gov.au

1 Microbiology and Immunology Section, Elizabeth Macarthur Agricultural Institute, Camden, NSW 2570, Australia

2 Department of Biotechnology, University of New South Wales, Sydney, NSW, Australia
Mycoplasma hyopneumoniae, $\boldsymbol{M}$. hyorhinis and $\boldsymbol{M}$. flocculare are commonly isolated from the respiratory tract of pigs and are phylogenetically related. The identification and characterization of antigens specific for $M$. hyopneumoniae is crucial for the development of serological reagents and for understanding the mechanisms of pathogenicity of this pathogen. Protein and antigen profiles of six strains of $M$. hyopneumoniae, four strains of $\boldsymbol{M}$. hyorhinis and a type strain of $\boldsymbol{M}$. flocculare were compared using SDS-PAGE and immunoblotting. Five strains of $\boldsymbol{M}$. hyopneumoniae originally isolated from diverse geographical regions produced similar protein and antigen profiles. One strain, C1735/2, produced a unique protein profile and was poorly immunoreactive, suggesting that some strains of $M$. hyopneumoniae may possess a structurally modified repertoire of antigens. Major $M$. hyopneumoniae antigens with molecular masses of approximately $36,43,48$, $52,76,78,80,82,94,106,114$ and $200 \mathrm{kDa}$ were identified by immunoblotting using hyperimmune pig sera raised against both high and low passage strains of $M$. hyopneumoniae. Porcine hyperimmune sera raised against the GDL type strain of $M$. hyorhinis reacted strongly with all $M$. hyorhinis strains although the profiles displayed considerable variation. Major antigens of molecular mass 42, 49, 52, 78, 80 and 82 kDa were identified in type strains GDL and BTS7 and field strain 2; however, field strain 1 produced a unique profile. A preparative SDS-PAGE profiling (PPP) technique was developed which enabled quantification of the immunoreactivity of denatured antigens with porcine serum by ELISA. PPP facilitated the rapid identification of species-specific and cross-reactive antigens among the three mycoplasma species. PPP studies revealed several strongly immunoreactive $M$. hyopneumoniae-specific antigens of 43, 76, 94, 114 and $200 \mathrm{kDa}$ as well as antigens of molecular mass between 52 and $62 \mathrm{kDa}$ which were not apparent in immunoblotting studies. Rabbit monospecific anti-43 kDa serum reacted specifically with a 43 kDa antigen in whole cell lysates of geographically diverse strains of $M$. hyopneumoniae and failed to cross-react with $M$. flocculare or $M$. hyorhinis whole cell lysates. This study has identified a number of $\boldsymbol{M}$. hyopneumoniaespecific antigens which warrant further investigation to determine their potential as diagnostic reagents and the role they play, if any, in pathogenicity.

Keywords: Mycoplasma byopneumoniae, Mycoplasma hyorbinis, diagnostic antigens, preparative SDS-PAGE profiling, immunoblotting 


\section{INTRODUCTION}

Mycoplasma byopneumoniae, M. byorhinis and $M$. flocculare are commonly found in the porcine respiratory tract. M. byopneumoniae is the cause of porcine enzootic pneumonia (PEP), which imposes a severe economic burden on swine production. $M$. hyorhinis has been implicated as an agent of arthritis and polyserositis (Ross, 1992; Friis \& Feenstra, 1994) whilst $M$. flocculare is generally regarded as non-pathogenic (Strasser et al., 1992). DNA-DNA hybridization studies and 16S rRNA sequence analysis have demonstrated that these porcine mycoplasma species exhibit phylogenetic similarities and form a sub-cluster within the fermentans phylogenetic group (Stemke et al., 1985, 1992). Further, more than $95 \%$ sequence homology exists between the 16S RNA sequences of $M$. byopneumoniae and $M$. flocculare, indicating a close phylogenetic relationship between these species (Stemke et al., 1992).

Serological cross-reactivity can be demonstrated between these three species using a variety of techniques including growth and metabolic inhibition assays (Friis, 1971), immunoelectrophoresis (Ro \& Ross, 1983), complement fixation (Freeman et al., 1984), immunoblotting (Bolske et al., 1987; Young \& Ross, 1987; Mori et al., 1988; Thirkell et al., 1991; Strasser et al., 1992) and ELISA (Freeman et al., 1984). The majority of these studies have focussed on antigenic differences between laboratory-adapted type strains of these three species. Earlier studies using growth and metabolic inhibition assays and the complement fixation test were unable to detect serological differences between the J type strain and several field strains of M. byopneumoniae (Roberts \& Little, 1970; Rose et al., 1979). Ro \& Ross (1983) compared eight field strains of M. byopneumoniae by two-dimensional immunoelectrophoresis and noted some antigenic diversity but were unable to define the molecular masses of similar and variant antigens. Thus, little is known about the molecular identity of antigens amongst strains isolated from different geographical locations and maintained in different laboratories.

Several studies have demonstrated that increasing in vitro passage reduces the virulence of $M$. byopneumoniae. Experimental challenge of pigs with low passage strains of $M$. hyopneumoniae induces lung lesions whilst inoculation with high passage $\mathrm{J}$ strain failed to cause disease (Tajima \& Yagihashi, 1982; Zielinski \& Ross, 1990). Low passage strains of $M$. byopneumoniae specifically attach to cilia in porcine tracheal organ cultures inducing ciliostasis and loss of cilia, but attempts to reproduce these pathogenic effects using strains of $M$. byopneumoniae which had undergone increasing in vitro passage either failed or were significantly diminished (DeBey \& Ross, 1994). Furthermore, Ross \& Young (1993) found that hyperimmune serum raised against a low passage virulent strain of $M$. hyopneumoniae, preabsorbed against a high passage avirulent strain, contained antibodies predominantly directed against several antigens with molecular masses between 46 and $145 \mathrm{kDa}$. These antigens, which were specific to or more abundant in the low passage strain, may be important virulence antigens. Such studies suggest that strains of $M$. hyopneumoniae varying in their degree of laboratory passage may display a variable antigenic repertoire.

We have previously described an ELISA, based on a $43 \mathrm{kDa}$ antigen, that detected serum antibodies against M. hyopneumoniae with a high sensitivity and specificity in naturally and experimentally infected pigs. This antigen was shown to be highly immunoreactive against porcine hyperimmune serum raised against $M$. hyopneumoniae and did not cross-react with anti-M. byorhinis and anti-M. flocculare sera (Djordjevic et al., 1994).

The aim of this study was to identify the repertoire of antigens amongst a small group of geographically diverse strains of $M$. byopneumoniae using anti- $M$. byopneumoniae antisera raised against both high and low passage strains of $M$. byopneumoniae by SDSPAGE and immunoblotting. In addition, anti- $M$. byopneumoniae, anti- $M$. byorbinis and anti-M. flocculare sera were used to determine the molecular masses of cross-reactive antigens between these three mycoplasma species and identify $M$. byopneumoniaespecific antigens. Furthermore, we developed and evaluated a preparative SDS-PAGE profiling (PPP) technique which combined the advantages of the electrophoretic separation of proteins with the convenience and speed of ELISA to identify species-specific and cross-reactive antigens. PPP offered the potential to quantify the humoral immune response against the entire repertoire of mycoplasmal antigens, facilitating the identification of highly immunoreactive and speciesspecific serological reagents. Finally, we evaluated the diagnostic utility of monospecific antiserum raised to a $43 \mathrm{kDa}$ antigen against whole cell lysates of strains of these three species of porcine mycoplasma by immunoblotting.

\section{METHODS}

Growth, preparation and identification of mycoplasma strains. The mycoplasma strains used in this study (Table 1) were obtained from the Australian Mycoplasma Reference Collection (AMRC), South Australian Department of Primary Industries, Central Veterinary Laboratory, Adelaide, Australia. Methods used to culture porcine mycoplasmas have been described by Sheldrake \& Romalis (1992). Briefly, both field (low passage) and laboratory adapted (high passage) strains of $M$. byopneumoniae, $M$. hyorhinis and $M$. flocculare were grown in modified Friis medium at $37^{\circ} \mathrm{C}$ and harvested during the exponential phase when the medium became honey coloured and $\mathrm{pH}$ was in the range $7 \cdot 2-6 \cdot 9$. Mycoplasma cells were harvested by centrifugation at $7000 \mathrm{~g}$ for $20 \mathrm{~min}$ and the pellet was washed three times with Tris-buffered saline (TBS: $10 \mathrm{mM}$ Tris/ $\mathrm{HCl}, 0.9 \mathrm{M} \mathrm{NaCl}$; $\mathrm{pH} \mathrm{7.4).} \mathrm{Mycoplasma} \mathrm{field}$ isolates had been originally typed using immunodiffusion (Taylor-Robinson et al., 1963) and growth-inhibition tests (Clyde, 1964) prior to storage in the AMRC. The identity of all strains of $M$. byopneumoniae and $M$. flocculare type strain $\mathrm{Ms} 42$ was confirmed using species-specific PCR primers 
Table 1. Mycoplasma strains used in this study

\begin{tabular}{|c|c|c|}
\hline Species & Strain & Source* \\
\hline M. byopneumoniae & J (NCTC 10110) & A. Pointon \\
\hline M. byopneumoniae & 232 & $\begin{array}{l}\text { T. Young } \\
\text { (Bereiter et al., } \\
\text { 1990) }\end{array}$ \\
\hline M. hyopneumoniae & $\mathrm{YZ}$ & M. Kobisch \\
\hline M. hyopneumoniae & Beaufort & AMRC \\
\hline M. byopneumoniae & Sue & AMRC \\
\hline M. hyopneumoniae & $\mathrm{C} 1735 / 2$ & AMRC \\
\hline M. hyopneumoniae & OMZ407 & AMRC \\
\hline M. flocculare & $\mathrm{Ms} 42$ & AMRC \\
\hline M. byorhinis & Field strain 2 & AMRC \\
\hline M. hyorhinis & BTS-7 & AMRC \\
\hline M. byorbinis & GDL & AMRC \\
\hline M. byorhinis & Field strain 1 & AMRC \\
\hline
\end{tabular}

* A. Pointon, South Australian Research \& Development Institute, Adelaide, Australia ; M. Kobisch, CNEVA PLOUFRAGAN, Station de Pathologie Porcine, Ploufragan, France; AMRC, Australian Mycoplasma Reference Collection.

(Stemke et al., 1994) which amplified portions of the $16 \mathrm{~S}$ rRNA gene of these two species.

Preparation of sera. Porcine hyperimmune sera were prepared against $M$. byopneumoniae J strain, $M$. byopneumoniae Beaufort strain, $M$. hyorbinis GDL strain and $M$. flocculare Ms42 strain as described by Sheldrake \& Romalis (1992). Briefly, these strains were freshly grown, washed and harvested as described. Each strain of mycoplasma $(1.0 \mathrm{~g}$ cell pellet, wet weight) was emulsified in Freund's complete adjuvant (total vaccine volume $2 \mathrm{ml}$ ) and $1 \mathrm{ml}$ was administered in the neck and rump of each of two pigs for each strain of mycoplasma. One month later, $2 \mathrm{ml}$ of a booster vaccine was prepared and administered as before (except that Freund's incomplete adjuvant was used). Animals were bled before vaccination (prebleed) and weekly after the second vaccination and the serum antibody response was monitored by immunoblotting and ELISA. Pigs were killed 4-6 weeks after the second vaccination and the serum was collected and stored at $-20^{\circ} \mathrm{C}$. Although minor variations in immunoblotting profiles were observed using sera collected from each animal vaccinated with the same antigen, antiserum that recognized the greatest number of antigens (determined by immunoblotting) was chosen for these studies.

Rabbit (New Zealand White) polyclonal antiserum raised against $M$. byopneumoniae strain $C 1735 / 2$ was prepared as described by Harlow \& Lane (1988). Briefly, two rabbits were vaccinated on two occasions (one month apart) each with $3.5 \mathrm{mg}$ of $\mathrm{C} 1735 / 2$ whole cells emulsified with Freund's complete adjuvant $(2 \mathrm{ml}$ total volume), injecting approximately $0.75 \mathrm{ml}$ intramuscularly into each of three limbs (both hindlimbs and one forelimb) on each occasion. The second vaccination was identical to the first except that Freund's incomplete adjuvant was used. The serum anti-M. byopneumoniae response was monitored weekly by immunoblotting and ELISA and the animals were exsanguinated 1 month after the booster vaccination. The serum was collected and stored at $-20^{\circ} \mathrm{C}$.
Preparation of SDSPAGE columns. Mycoplasma cells (50$100 \mathrm{mg}$ in $2 \mathrm{ml}$ ) were reduced in Laemmli mixture by boiling (Laemmli, 1970) and several crystals of bromophenol blue were added to dye the sample. Cell preparations were loaded onto either $5 \%$ or $9 \%$ polyacrylamide gel columns (Bioprep 491 cell, Bio-Rad), $8 \mathrm{~cm}$ or $12 \mathrm{~cm}$ in height respectively with a $1 \mathrm{~cm}$ stacking gel consisting of $4 \%$ polyacrylamide. Identical volumes (i.e. identical column heights) were used for the preparation of all polyacrylamide columns. Protein concentration was estimated using a BCA protein test kit (Pierce).

Columns were run at $40 \mathrm{~mA}$ constant current $(150-300 \mathrm{~V})$ for $24 \mathrm{~h}$ and fractions $(9.5 \mathrm{ml})$ were collected immediately after elution of the bromophenol blue dye. The electrophoresis and elution buffers comprised SDS-PAGE buffer (Towbin et al., 1979) and the flow rate was $0.53 \mathrm{ml} \mathrm{min}{ }^{-1}$. Fractions were stored at $4{ }^{\circ} \mathrm{C}$ for several weeks or at $-20^{\circ} \mathrm{C}$ for up to one year. Protein profiles detected either by SDS-PAGE or ELISA remained unchanged throughout storage. Molecular masses of peak fractions were estimated by SDS-PAGE and silver staining with known commercial molecular size markers.

SDSPAGE and immunoblotting analysis. Column fractions (undiluted $30 \mu \mathrm{l}$ aliquots from $9.5 \mathrm{ml}$ fractions) were analysed by one-dimensional polyacrylamide gel electrophoresis under reducing conditions (Laemmli, 1970) in a Hoeffer mini-gel system. Protein samples were heated to $95{ }^{\circ} \mathrm{C}$ for $5 \mathrm{~min}$ immediately prior to loading onto a $10 \%$ polyacrylamide resolving gel containing a $4 \%$ stacking gel. Electrophoresis was performed with constant current $(20 \mathrm{~mA})$ until the tracking dye (bromophenol blue) had migrated to the bottom of the resolving gel. Polyacrylamide gels were stained with either silver or Coomassie blue. Western blots were performed using a Hoeffer Trans-Blot as described previously (Djordjevic et al., 1994). Molecular masses were calculated using prestained and unstained low range markers (Bio-Rad). Immunoblots were blocked using $5 \%$ skim milk in TBS $(10 \mathrm{mM}$ Tris/ $\mathrm{HCl}, 0.9 \mathrm{M} \mathrm{NaCl} ; \mathrm{pH} 7.4)$, reacted with porcine hyperimmune sera $(1: 500$ in TBS with $0.1 \%$ skim milk) and incubated with slow shaking at room temperature for $1.5 \mathrm{~h}$. Immunoblots were reacted $(1 \mathrm{~h})$ with goat anti-swine $\mathrm{Ig}$ $(\mathrm{H}+\mathrm{L})$ horseradish peroxidase conjugate (Southern Biotechnics) $(1: 1000$ in TBS with $0 \cdot 1 \%$ skim milk). Blots were washed $(3 \times 5 \mathrm{~min})$ in Tris $\mathrm{HCl}(100 \mathrm{mM}, \mathrm{pH} 7.6)$ prior to colour development with 3,3'-diaminobenzidine tetrahydrochloride (DAB) $(0.05 \%$ in Tris $/ \mathrm{HCl}, 100 \mathrm{mM}, \mathrm{pH} 7.6)$.

ELISA. Aliquots $(10 \mu \mathrm{l})$ of the column fractions were loaded into 96-well ELISA plates (Nunc) and diluted 1:10 with carbonate loading buffer ( $30 \mathrm{mM}$ sodium carbonate, $20 \mathrm{mM}$ sodium hydrogen carbonate, $\mathrm{pH} 9.6$ ) and left overnight in a humid chamber at $21^{\circ} \mathrm{C}$. Plates were washed $(5 \times 400 \mu \mathrm{l})$ with TW (Milli Q water $/ 0.05 \%$ Tween 20) using a Titertek microplate washer. Primary antibody (hyperimmune sera against $M$. hyopneumoniae strains J, Beaufort, M. hyorbinis strain GDL and M. flocculare strain Ms42) was diluted 1:200 in $10 \%$ normal goat serum in PBST (phosphate buffered saline, $\mathrm{pH} 7.2$ containing $0.05 \%$ Tween 20 ) and $100 \mu \mathrm{l}$ was added to the wells. Plates were incubated for $2 \mathrm{~h}$ at $21^{\circ} \mathrm{C}$ and washed $(5 \times 400 \mu \mathrm{l})$ as described above. Horseradishperoxidase-conjugated goat anti-swine $\mathrm{Ig}(\mathrm{H}+\mathrm{L}$ ) (Southern Biotechnics, $100 \mu \mathrm{l}$ ) diluted $1: 1000$ in $10 \%$ normal goat serum in PBST was added and the plates incubated at room temperature for $1 \mathrm{~h}$. After a final washing step $(5 \times 400 \mu \mathrm{l})$ colour development was achieved by the addition of $100 \mu \mathrm{l}$ of $1 \mathrm{mM} 2,2^{\prime}$-azinodiethylbenzthiazolinsulfonate (ABTS) diluted in citrate/phosphate buffer $(\mathrm{pH} \mathrm{4.2)}$ and incubated at room temperature until the desired colour developed. Absorbance was read at $405 \mathrm{~nm}$ (Titertek plate reader) when the colour 


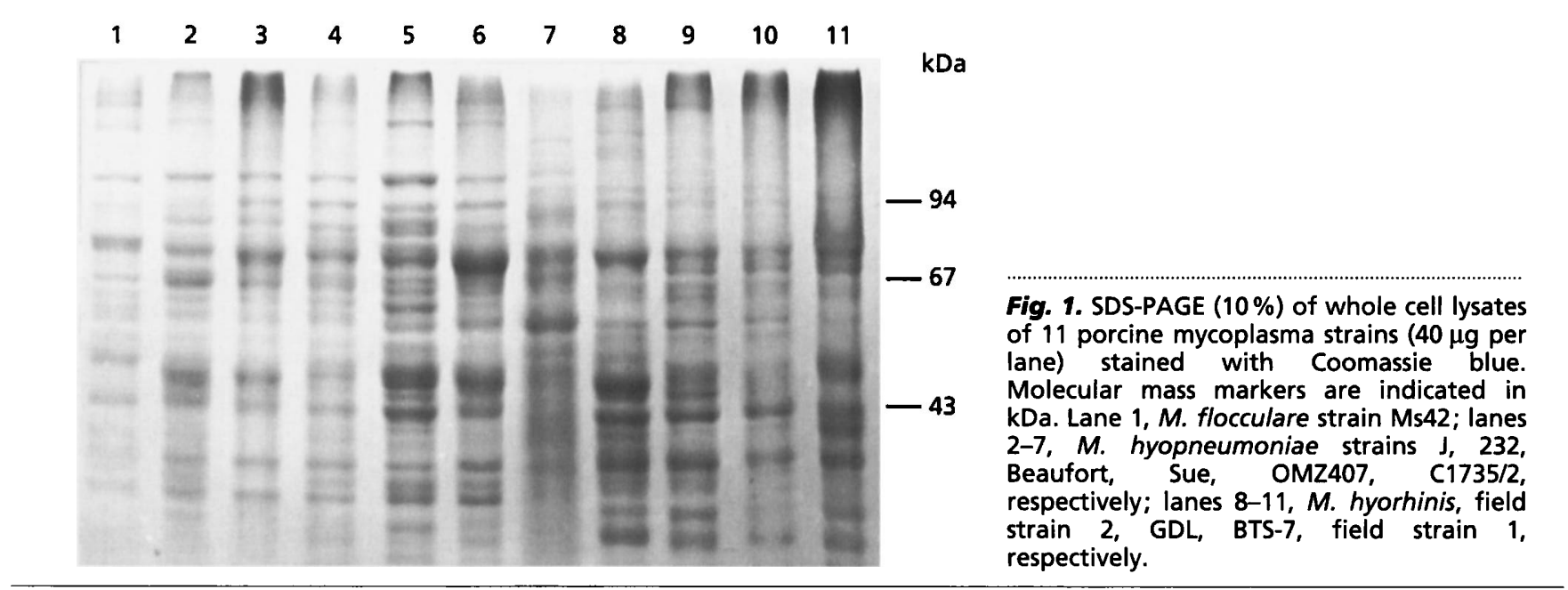

development reached an absorbance for fractions containing the $43 \mathrm{kDa}$ antigen approximately 10 -fold higher than fractions containing only electrophoresis buffer (negative control). Anti-swine HRP conjugate did not cross-react with any PPP fraction in control experiments.

\section{RESULTS}

\section{Whole cell protein analysis by SDSPAGE}

Whole cell extracts of $M$. hyopneumoniae, M. hyorbinis and $M$. flocculare were resolved by SDS-PAGE and the proteins stained with Coomassie blue. Electrophoretic profiles of six strains of $M$. hyopneumoniae, four strains of $M$. byorbinis and a type strain of $M$. flocculare are shown in Fig. 1. M. byopneumoniae strains J and 232 (both high passage laboratory adapted strains), Sue, Beaufort and OMZ407 (low passage strains) had very similar protein profiles (although none of the profiles were identical), with some variation evident in the region between 80 nd $85 \mathrm{kDa}$. M. hyopneumoniae strain C1735/2 (low passage field strain) showed considerably greater variation compared with the other five strains, particularly in the $80-140 \mathrm{kDa}$ region. $M$. flocculare type strain Ms42 produced a unique electrophoretic profile; however, many bands aligned at similar molecular sizes with those typical of $M$. byopneumoniae. All four $M$. hyorhinis strains produced very similar electrophoretic profiles and were easily distinguished from the profiles representative of the other two mycoplasma species.

\section{Antigenic analysis by immunoblotting}

Homologous immunoreactivity. Immunoblot analysis using anti-M. hyopneumoniae hyperimmune serum prepared against a high passage, laboratory adapted strain J (Fig. 2a) and a low passage $(<5)$ Beaufort isolate (Fig. $2 b$ ) were used to probe whole cell extracts of the 11 porcine mycoplasmas described in Table $1 . M$. byopneumoniae strains J, 232, Sue, Beaufort and OMZ407 produced similar profiles using both hyperimmune sera. Hyperimmune sera raised against the $\mathrm{J}$ strain produced immunoreactive bands with approxi- mate molecular masses at $36,43,48,52,76,78,80,82,94$ and $114 \mathrm{kDa}$ (Fig. 2a, lane 1). Some M. hyopneumoniae strains lacked some of these immunoreactive bands.

Hyperimmune serum raised against the Beaufort strain identified antigens of approximate molecular mass $30(\mathrm{~J}$ strain only), 36-38, 42 (weak), 43, 48, 65 (weak), 72 (weak), 76, 78, 80-82, 94, 106, 114, $200 \mathrm{kDa}$ and a band greater than $200 \mathrm{kDa}$ (Fig. 2b). Immunoreactivity against antigens of molecular mass greater than $85 \mathrm{kDa}$ (particularly 94, 106, $114 \mathrm{kDa}$ antigens) was more intense using the anti-Beaufort hyperimmune serum. Immunoreactive $M$. byopneumoniae antigens in the molecular mass range of $34-36,94,106$ and $114 \mathrm{kDa}$ showed minor intra-species size variation; however, immunoblots probed with monoclonal or monospecific polyclonal antibodies against these antigens would be required to confirm these observations.

Antigens of $M$. byopneumoniae strain $\mathrm{C} 1735 / 2$ reacted poorly with both hyperimmune anti-M. hyopneumoniae sera; these sera identified antigens with molecular masses of 43, 46-48 (weak), 78 and (anti-Beaufort antiserum only) $80 \mathrm{kDa}$ (Fig. 2a, b). This antigen profile was more similar to the profile generated by whole cell antigens of $M$. flocculare strain Ms42. In an attempt to further characterize the antigen profile of strain $\mathrm{C} 1735 / 2$, rabbit hyperimmune anti-C1735/2 antiserum was raised and used to probe an immunoblot containing whole cell antigens of the six $M$. byopneumoniae strains. A typical $M$. hyopneumoniae antigen profile showing antigens with approximate molecular masses of 36,43 , $48-52,76,78,94$ and $114 \mathrm{kDa}$ was observed for all $M$. byopneumoniae strains except $\mathrm{C} 1735 / 2$, which produced a smearing pattern without clearly defined banding (data not shown).

Heterologous immunoreactivity. M. flocculare antigens of molecular masses 43,55 and $78 \mathrm{kDa}$ cross-reacted with hyperimmune antisera raised against both the $\mathrm{J}$ and Beaufort strains (Fig. 2a and b, respectively, lanes 8) of M. hyopneumoniae. M. flocculare antigens of molecular masses 76, 114 and $200 \mathrm{kDa}$ reacted faintly with the anti-Beaufort antiserum. $M$. byorhinis antigens also 
(a)

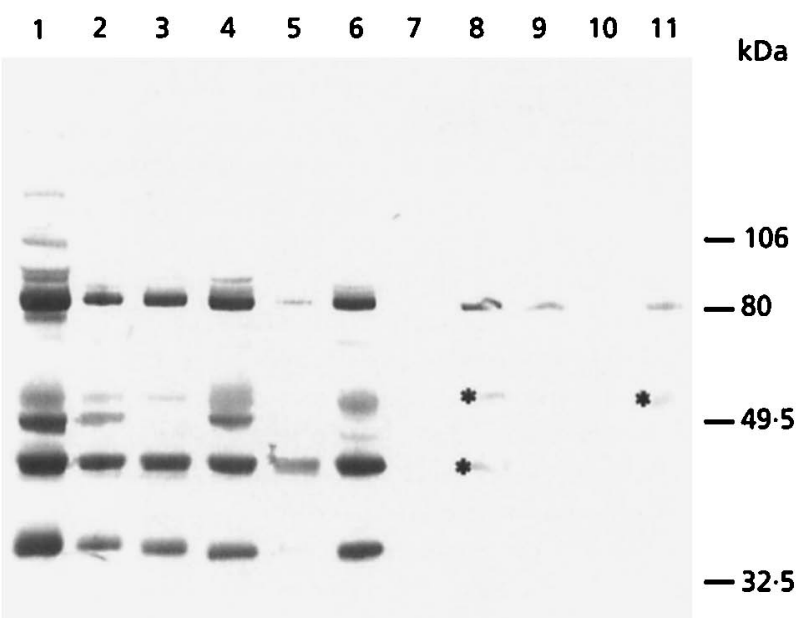

(b)

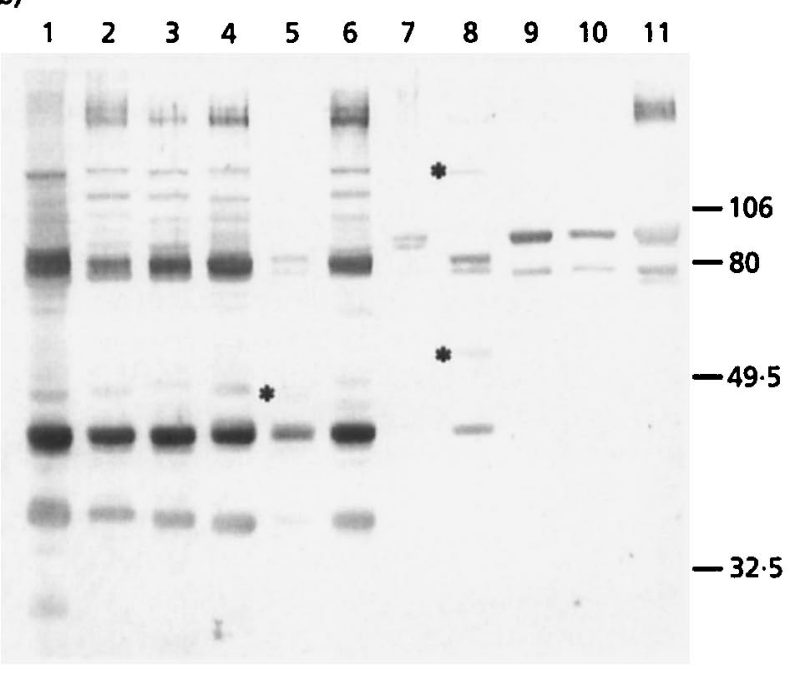

(c)

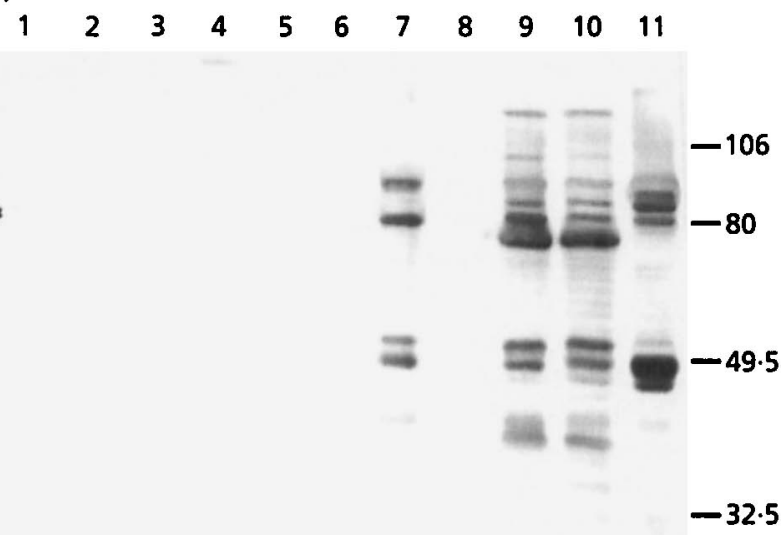

Fig. 2. Immunoblot analysis of 11 porcine mycoplasma strains. Whole cell lysates $(20 \mu \mathrm{g}$ per lane) were resolved on $10 \%$ SDSpolyacrylamide gels, transferred onto PVDF membrane and reacted with antisera raised against $M$. hyopneumoniae $J$ strain cross-reacted with both anti- $M$. hyopneumoniae sera recognizing $78 \mathrm{kDa}$ antigens, and $85 \mathrm{kDa}$ and approximately $200 \mathrm{kDa}$ antigens reacting only with the antiBeaufort antiserum. Pig anti-M. flocculare sera crossreacted weakly with $M$. hyopneumoniae antigens of molecular masses $25,28,48 \mathrm{kDa}$ and more strongly with a $52 \mathrm{kDa}$ antigen (data not shown). Anti-M. flocculare sera also detected cross-reactive $M$. hyorbinis antigens of 48,52 (strong) and 114 (extremely faint) $\mathrm{kDa}$ (data not shown).

\section{Immunoreactive antigen profiles using porcine anti- $M$. hyorhinis hyperimmune serum}

Fig. 2(c) shows an immunoblot containing whole cell mycoplasma lysates reacted with hyperimmune sera raised against $M$. hyorhinis type strain GDL. Strongly immunoreactive $M$. byorbinis antigens were identified at $42,49,52,78,80$ and $82 \mathrm{kDa}$. Weakly immunoreactive antigens were observed at 36, 44, 47, 85, 94, 106 and $114 \mathrm{kDa}$. This antigen profile was identical for both type strains GDL and BTS-7 (except for the $36 \mathrm{kDa}$ antigen, which was identified only in strain BTS-7). M. hyorhinis field strain 2 displayed strongly immunoreactive antigens of molecular masses $49,52,80$, and $85 \mathrm{kDa}$ and weakly immunoreactive antigens of 44, 82, 84 and $114 \mathrm{kDa}$ showing similarity with profiles typical of $M$. hyorhinis type strains BTS-7 and GDL. M. hyorhinis field strain 1 displayed strongly immunoreactive antigens of molecular masses $47,49,80,82$, and $83 \mathrm{kDa}$ and weakly immunoreactive antigens of molecular masses 52 and $85 \mathrm{kDa}$, producing a unique profile. Although the four strains of $M$. hyorhinis display very similar protein profiles (Fig. 1) and cross-reactive antigen profiles with anti-M. byopneumoniae sera (Fig. $2 a, b)$, considerable antigenic heterogeneity is evident using anti-M. hyorhinis serum. Similar observations have been described for many other species of mycoplasma (Andersen et al., 1987; Städtlander et al., 1991; Poumarat et al., 1994). Faintly immunoreactive antigens ranging in molecular mass from 50 to $78 \mathrm{kDa}$ and differing in molecular size by approximately $2 \mathrm{kDa}$ are evident in lanes containing all four $M$. hyorbinis strains (Fig. 2c). Further studies are required to determine the degree and significance of antigenic variability amongst strains of $M$. hyorbinis.

$M$. hyopneumoniae strains and $M$. flocculare type strain Ms42 failed to cross-react strongly with the anti-M. byorhinis serum (Fig. 2c). An extremely weak cross-

(a), $M$. hyopneumoniae Beaufort strain (b), and $M$. hyorhinis GDL strain (c). For all blots: lanes $1-6, M$. hyopneumoniae strains J, 232, Beaufort, Sue, C1735/2, OMZ407, respectively; lane 7, $M$. hyorhinis field strain 2; lane 8, $M$. flocculare Ms42; lanes 9-11, $M$. hyorhinis GDL, BTS-7, field strain 1, respectively. Asterisks identify the presence of faintly immunoreactive bands. An extremely faint cross-reactive $M$. hyopneumoniae antigen of $81 \mathrm{kDa}$ is present in all six strains, but can only be observed in lanes 1 and 2 in (c), which contain whole cell lysates of $M$. hyopneumoniae strains $J$ and 232. 
(a)

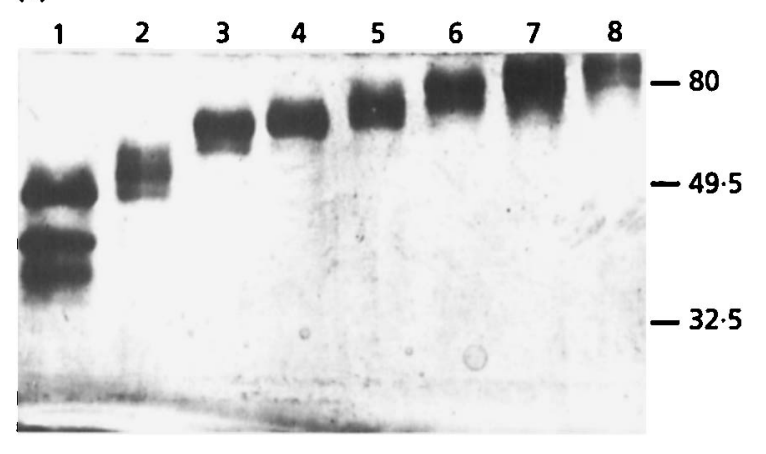

(b)

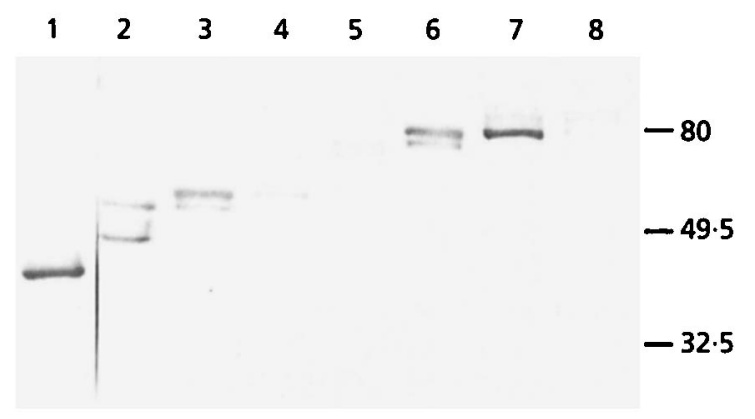

Fig. 3. (a) Silver-stained $10 \%$ SDS-polyacrylamide gel of every third fraction eluted from a $9 \%$ SDS-polyacrylamide column. Fractions contained one to three bands. (b) Immunoblot of the same fractions eluted from a $9 \%$ SDS-polyacrylamide column resolved on a $10 \%$ SDS-polyacrylamide slab gel, transferred onto PVDF membrane and reacted with antisera raised against $M$. hyopneumoniae J strain.

reactive antigen $(81 \mathrm{kDa})$ was observed in all $M$. byopneumoniae strains.

\section{Preparative SDSPAGE profiling (PPP)}

The BioPrep 491 SDS-PAGE column has been used previously for the electrophoretic separation and purification of preparative quantities of $M$. byopneumoniae membrane proteins (Djordjevic et al., 1994). Preliminary investigations showed that $9 \%$ polyacrylamide columns adequately separated antigens within a molecular mass range of $20-80 \mathrm{kDa}$ and a $5 \%$ polyacrylamide column resolved high molecular mass antigens between 80 and $250 \mathrm{kDa}$. Fig. 3(a, b) shows that fractions $(9.5 \mathrm{ml})$ eluted from a $9 \%$ SDS-PAGE column contained between one and three discernible antigens when resolved on a $10 \%$ polyacrylamide mini-gel stained with silver (Fig. 3a) or immunoblotted with hyperimmune serum against $M$. byopneumoniae strain J (Fig. 3b). Analytical columns (28 $\mathrm{mm}$ internal diameter) produced well-resolved antigen profiles when loaded with approximately $50-100 \mathrm{mg}$ of denatured mycoplasma whole cell antigen. Higher loading concentrations reduced the resolving capabilities of the column.

In an attempt to quantify the immunoreactivity of $M$. byopneumoniae antigens we investigated the possibility of adsorbing aliquots from each fraction onto 96-well ELISA plates and probing the antigens with anti- $M$. byopneumoniae hyperimmune serum. Preliminary experiments with titrated $M$. hyopneumoniae whole cell lysate (prepared by boiling with reducing solution) bound to ELISA plates and reacted with hyperimmune serum revealed a linear relationship between antigen concentration and ELISA reactivity as measured by absorbance (data not shown). A M. byopneumoniae strain J profile representative of the first 50 fractions which eluted from a $9 \%$ polyacrylamide column and reacted with hyperimmune serum raised to $M$. byopneumoniae strain J is shown in Fig. 4(a). Immunoreactive peaks were evident in fractions 5 and 6,9 and $10,12-17,21-33$ and $40-47$ which corresponded to molecular masses of approximately $36,43,48-62,65-83$ and $94 \mathrm{kDa}$. Molecular masses of antigens in peak fractions were determined using commercial molecular mass markers on SDS-polyacrylamide mini-gels stained with silver. The majority of these immunoreactive ELISA peaks were also detected on immunoblots (Fig. 2). Notable exceptions include the ELISA reactive antigens with molecular masses between 52 and $62 \mathrm{kDa}$, which were absent or appeared as extremely faint bands on immunoblots (Fig. 2a, b). Fig. 4(b) shows an SDSPAGE ELISA profile of a $5 \%$ polyacrylamide column loaded with whole cell lysate of the J strain of $M$. hyopneumoniae. Immunoreactive peaks of molecular sizes $94,106,114$ and $200 \mathrm{kDa}$ are well resolved. These antigens were also detected by immunoblotting (Fig. 2), although the $200 \mathrm{kDa}$ antigen is not usualy recognized with hyperimmune sera raised against the J strain of $M$. byopneumoniae.

\section{Comparison of PPP of $M$. hyopneumoniae, $M$. hyorhinis and $M$. flocculare type strains against anti-M. hyopneumoniae J strain serum}

Fig. 5 shows PPP, eluted from $9 \%$ (Fig. 5a) and $5 \%$ (Fig. $5 \mathrm{~b}$ ) polyacrylamide columns, respectively, of antigens of the three porcine mycoplasma type strains reacted with hyperimmune sera raised against the $J$ strain of $M$. byopneumoniae. Strongly immunoreactive $M$. hyopneumoniae antigens were identified with molecular masses of 43, 76, 94, 114 and $200 \mathrm{kDa}$ which appeared specific for $M$. hyopneumoniae. Strong cross-reactivity was observed with $M$. flocculare antigens of molecular masses 36,55 and $78 \mathrm{kDa}$. Interestingly, cross-reactivity of the $36 \mathrm{kDa}$ antigen was not observed by immunoblotting (Fig. 2). Weak cross-reactivity was observed with $M$. hyorhinis antigens of molecular masses 55 and $78 \mathrm{kDa}$. PPP generated from mycoplasma antigens eluted from $5 \%$ polyacrylamide columns using the antistrain J hyperimmune serum showed that the majority of the cross-reactivity was associated with the first three fractions (comprising antigens of molecular masses up to $80 \mathrm{kDa}$ ) which migrate just behind the dye front.

PPP studies using anti-M. hyorhinis serum identified cross-reactive $M$. hyopneumoniae J strain antigens of molecular masses $50-55,65$ and $74-78 \mathrm{kDa}$ (data not 


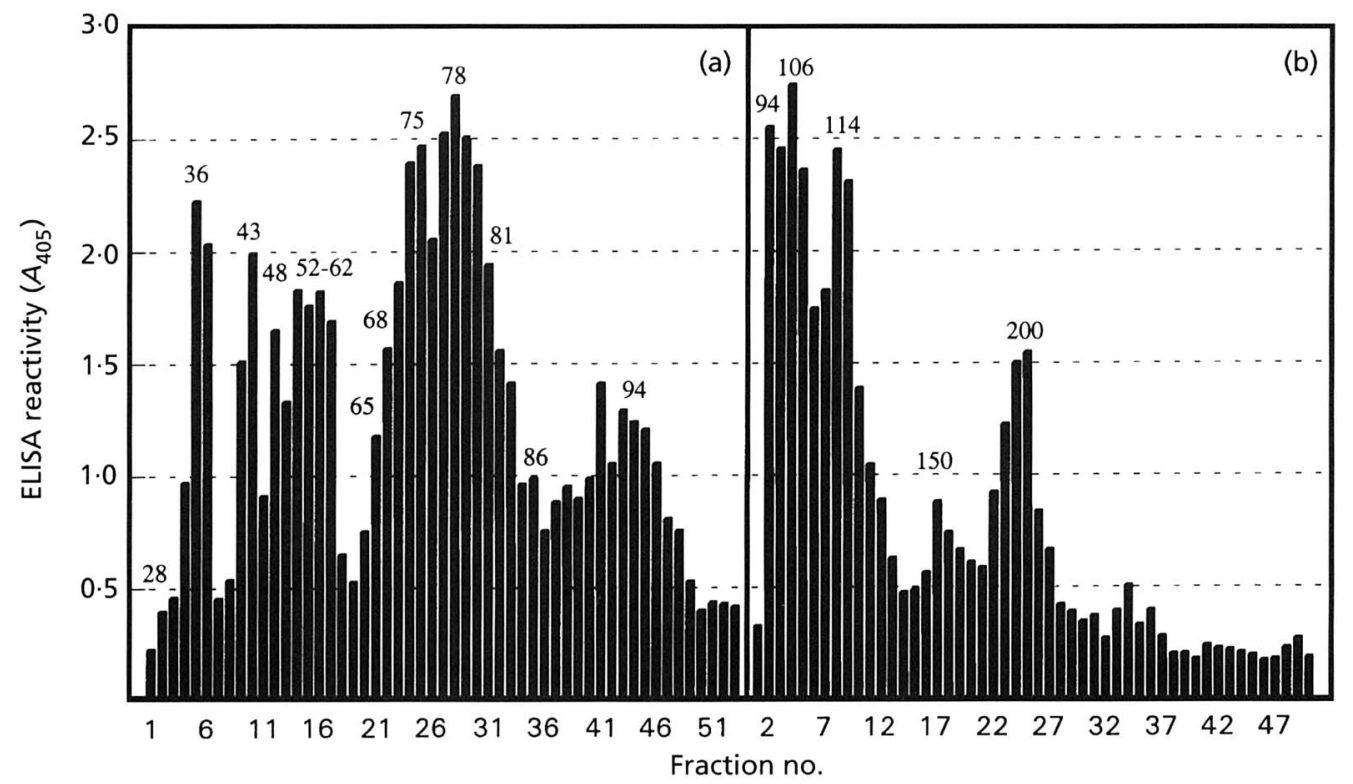

Fig. 4. PPP of $M$. hyopneumoniae $J$ strain whole cell lysate showing ELISA reactivity $\left(A_{405}\right)$ for each consecutive fraction that eluted from the column. Aliquots $(10 \mu \mathrm{l})$ of each fraction were coated onto a microtitre plate and reacted against hyperimmune serum raised to the J strain of $M$. hyopneumoniae. Panels (a) and (b) show PPP of the first 50 fractions eluted from $9 \%$ and $5 \%$ polyacrylamide columns respectively. The numbers on the profile indicate molecular masses $(\mathrm{kDa})$.

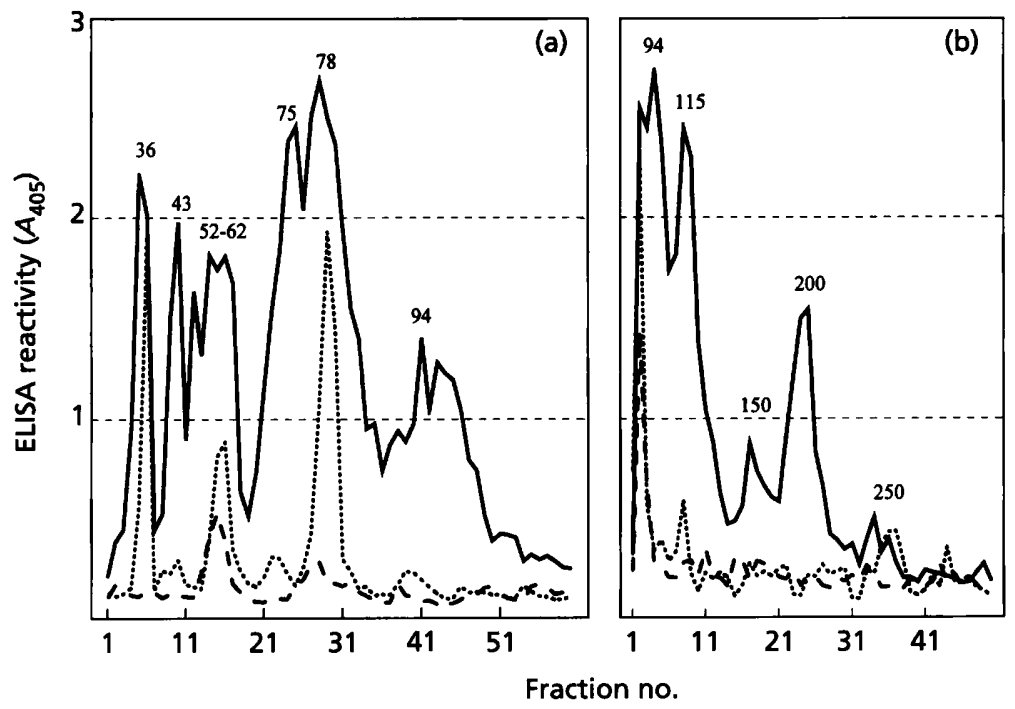

Fig. 5. Superimposed PPP of $9 \%($ a) and $5 \%$ (b) columns of whole cell lysates derived from type strains of $M$. hyopneumoniae (J, solid line), $M$. hyorhinis (GDL, dashed line), and $M$. flocculare (Ms42, dotted line) probed with hyperimmune serum raised against the $J$ strain of $M$. hyopneumoniae. ELISA reactivity $\left(A_{405}\right)$ was measured for each consecutive fraction that eluted from the column. Panels (a) and (b) show PPP of the first 50 fractions eluted from $9 \%$ and $5 \%$ polyacrylamide columns respectively. The numbers above the profile indicate molecular masses (kDa).

shown), whilst anti- $M$. flocculare serum identified weakly cross-reactive $M$. hyopneumoniae antigens with molecular masses of 50-55 and $65 \mathrm{kDa}$ (data not shown). The basis for the identification of species-specific antigens by the absence of cross-reacting antigens in other mycoplasma species is based on an assumption that cross-reactive antigens of different molecular masses are unrelated. Further studies are required to confirm the species specificity of these antigens.

\section{Purification and specificity of a $\mathbf{4 3} \mathrm{kDa}$ M. hyopneumoniae-specific antigen}

Although we have determined from immunoblotting and PPP studies that antigens of $36,43,76,94,114$ and $200 \mathrm{kDa}$ are specific for M. byopneumoniae, we chose one of these antigens to further evaluate its diagnostic potential. The $43 \mathrm{kDa}$ antigen was selected for two reasons: first, whilst PPP studies identified the $43 \mathrm{kDa}$ 


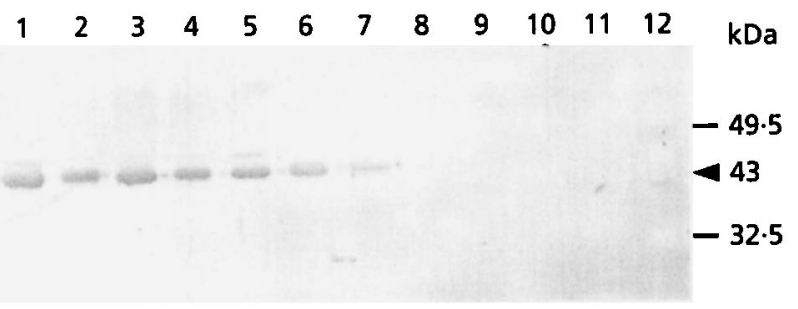

Fig. 6. Immunoblot analysis of 12 porcine mycoplasma strains using monospecific anti-43 kDa sera. Whole cell lysates $(20 \mu \mathrm{g}$ per lane) were resolved on $10 \%$ SDS-polyacrylamide gels, transferred onto PVDF membrane and reacted with rabbit anti$43 \mathrm{kDa}$ serum raised against a putative $M$. hyopneumoniaespecific $43 \mathrm{kDa}$ antigen. Lanes $1-7, M$. hyopneumoniae isolates J, 232, YZ, Beaufort, Sue, OMZ407, C1735/2, respectively; lane 8, MS42 ( $M$. flocculare); lanes 9-12, $M$. hyorhinis field strain 2, GDL, BTS-7, field strain 1, respectively.

antigen as an M. hyopneumoniae-specific antigen (Fig. 5), immunoblotting studies detected a cross-reactive $43 \mathrm{kDa}$ antigen in a whole cell lyste of $M$. flocculare using anti-M. hyopneumoniae serum (Fig. 2); and second, we had previously used the $43 \mathrm{kDa}$ antigen as an ELISA antigen to detect porcine serum antibodies against $M$. byopneumoniae but had not determined the presence of this antigen amongst geographically diverse field and laboratory-adapted strains of this pathogen. Fraction 9 (from a single column run) containing the $43 \mathrm{kDa}$ antigen was concentrated by ultrafiltration (Amicon, YM30) and emulsified with Freund's adjuvant for the generation of monospecific polyclonal antisera. Fig. 6 shows an immunoblot of whole cell lysates of 12 porcine mycoplasma strains reacted against rabbit anti$43 \mathrm{kDa}$ serum. M. hyopneumoniae strain YZ (originating in France) became available in the latter stages of this study and was included in this experiment to test the specificity of the anti- $43 \mathrm{kDa}$ antiserum against as many $M$. hyopneumoniae strains from different geographic origins as possible. A strongly reactive band of molecular mass $43 \mathrm{kDa}$ is present in the whole cell lysates of all $M$. hyopneumoniae strains but absent from lanes containing whole cell lysates of $\dot{M}$. hyorhinis and $M$. flocculare. A faintly reactive band of approximate molecular mass $45 \mathrm{kDa}$ is evident in lanes containing $M$. byopneumoniae strains J (lane 1) and Sue (Lane 5) and may be due to antigenic size variation in these two strains.

\section{DISCUSSION}

Protein and antigenic variation among mycoplasmas has traditionally been studied using SDS-PAGE and immunoblotting techniques. However, these techniques do not readily facilitate quantitative comparisons between strains unless densitometric analyses are performed. ELISA is one of the most sensitive, versatile and rapid diagnostic assay formats and has been used to detect and quantify the immune response against mycoplasmal antigens (Ross \& Young, 1993). SDS-PAGE, immunoblotting and various chromatographic matrices have been used to identify and recover diagnostic mycoplasmal antigens for ELISA (Sheldrake \& Romalis, 1992; Bolske et al., 1987; Young \& Ross, 1987; Mori et al., 1988; Kazama et al., 1989). Comparatively lengthy time periods are often required to run and monitor fractions eluted from chromatographic columns. Other limitations include difficulties with producing preparative quantities of desired antigen(s), and atypical chromatographic behaviour of some antigens in column matrices (Sheldrake \& Romalis, 1992). In this communication we report the development of a preparative SDS-PAGE profiling technique which can rapidly identify and quantify the immunoreactive responses against the spectrum of whole cell mycoplasmal proteins, facilitating the identification of potentially useful diagnostic antigens. Using hyperimmune sera to profile whole cell lysates of $M$. hyopneumoniae, $M$. byorbinis and $M$. flocculare, antigens of molecular masses $36,43,76,94,114$ and $200 \mathrm{kDa}$ were shown to be $M$. byopneumoniae-specific, highly immunoreactive antigens. One of these antigens $(43 \mathrm{kDa})$ was used to produce polyclonal antisera to confirm its specificity for M. hyopneumoniae in an immunoblot assay. Although the $43 \mathrm{kDa}$ antigen has been shown in a previous study to (i) fail to cross-react with porcine hyperimmune anti$M$. hyorbinis and anti-M. flocculare antisera and (ii) form the basis of a diagnostic ELISA for the detection of serum antibodies against $M$. byopneumoniae (Djordjevic et al., 1994), the utility of anti-43 kDa sera as a diagnostic antiserum for immunoblotting studies has not been addressed. Polyclonal antibodies raised against this antigen were able to detect a $43 \mathrm{kDa}$ band on immunoblots containing whole cell lysates of the seven strains of $M$. hyopneumoniae described in this study and failed to cross-react with whole cell lysates of M. flocculare and M. hyorbinis.

The presence of species-specific and cross-reactive antigens among type strains of $M$. byopneumoniae, $M$. hyorhinis and $M$. flocculare has been described previously in immunoblot studies (Bolske et al., 1987; Young \& Ross, 1987; Mori et al., 1988; Thirkell et al., 1991). Thirkell et al. (1991) described cross-reactive antigens between $M$. byopneumoniae and $M$. flocculare of molecular mass 52, 58, 64, 74, 107 and $184 \mathrm{kDa}$ whilst Young \& Ross (1987) identified cross-reactive antigens of 41, 50 and $110 \mathrm{kDa}$. Mori et al. (1988) identified crossreactive antigens in $M$. flocculare probed with anti-M. hyopneumoniae serum with molecular masses of 46 and $74 \mathrm{kDa}$. Similarly, Bolske et al. (1987) described a $73 \mathrm{kDa}$ antigen common to all three mycoplasma species and 41 and $35 \mathrm{kDa}$ antigens common to $M$. byopneumoniae and $M$. flocculare. In this study we identified cross-reactive antigens in $M$. flocculare with molecular masses of 36,55 and $78 \mathrm{kDa}$ in PPP studies (Fig. 5) and of 43,55 and $78 \mathrm{kDa}$ in immunoblotting studies using anti-M. hyopneumoniae sera (Fig. 2). Although it is feasible that the 36,43 and $78 \mathrm{kDa}$ antigens described here are similar to those described in other studies, further experiments are required to confirm this. The molecular masses of these antigens 
vary considerably between studies and this variation could be due to any one of a number of different experimental parameters including different sources (pig versus rabbit) and type of antisera (convalescent versus hyperimmune) and the different methods used to extract antigens. Strains of mycoplasma derived from various geographical locations with different degrees of in vitro passage could be expected to display different antigen profiles. In addition, minor differences in relative mobilities of antigens in different studies may be a function of the different molecular mass markers (stained versus unstained) used to estimate molecular mass. Finally, antigenic size variation has recently been described for an antigen from M. byopneumoniae with a molecular mass of $97 \mathrm{kDa}$ (Zhang et al., 1995) and this phenomenon is likely to alter the relative molecular masses of some antigens amongst different strains of this species.

Considerable genomic heterogeneity has been reported among strains of $M$. byopneumoniae recovered from different geographical locations in both Europe and the United States (Frey et al., 1992; Artiushin \& Minion, 1996) and considerably greater genomic variation was observed when comparing $M$. hyopneumoniae with $M$. flocculare (Frey et al., 1992; Chan \& Ross, 1984). Genomic heterogeneity is common among mycoplasma species and it is thought that mycoplasmas evolve rapidly (Razin et al., 1983; Christiansen \& Andersen, 1988; Ionas et al., 1991; Frey et al., 1992; Poumarat et al., 1994; Artiushin \& Minion, 1996). The fastidious nature of M. byopneumoniae, its slow growth and the complex medium requirements needed to culture field strains are reflected by the paucity of studies comparing protein and antigen profiles of this species. We examined six strains of $M$. byopneumoniae isolated from diverse geographical regions together with strains of $M$. byorbinis and $M$. flocculare using SDS-PAGE and immunoblotting techniques. The electrophoretic profiles produced by $M$. byopneumoniae strains J, Beaufort, Sue, 232 and OMZ407 are very similar. Strains $\mathrm{J}$ and 232 are high passage laboratory adapted strains originally isolated in Great Britain (Goodwin et al., 1965) and the United States (Bereiter et al., 1990) respectively, whilst OMZ407, Sue and Beaufort are field strains with a low passage history recovered from pneumonic lungs in pigs from Victoria, Australia. Only strain $\mathrm{C} 1735 / 2$ displayed a different protein profile compared with the other $M$. hyopneumoniae strains. Although the geographical origin of strain C1735/2 is unconfirmed the circumstances of its appearance in a PEP-free herd in Queensland, Australia, suggests that it may have originated from Canada (see below). One dimensional SDS-PAGE studies carried out on denatured whole cell lysates of other mycoplasmal species have not identified major intraspecies antigenic variation (Andersen et al., 1987; Städtlander \& Watson, 1992; Poumarat et al., 1994).

In contrast, considerable antigen profile variability has been reported amongst mycoplasmas belonging to the same species (Andersen et al., 1987; Stadtlander et al.,
1991; Avakian et al., 1991; Städtlander \& Watson, 1992). Poumarat et al. (1994) observed marked differences in the antigenic profiles amongst 20 Mycoplasma bovis field strains belonging to both the same and different genomic groups. However, all $M$. byopneumoniae strains reported in this communication (except $\mathrm{C} 1735 / 2$ ) produced very similar antigen profiles. The conservation of antigens amongst $M$. byopneumoniae strains and a relatively high degree of genomic heterogeneity suggest that these antigens are evolutionarily conserved and may function in crucial biological roles important for the survival of the species.

Unlike the similarity of antigen profiles among strains of $M$. hyopneumoniae, the profiles generated by anti- $M$. byorbinis antisera of the two field strains of $M$. hyorbinis were readily distinguishable from one another and from the profiles generated by type strains GDL and BTS-7. This result was unexpected as the electrophoretic profiles of whole cell lysates stained with Coomassie blue were very similar for all four $M$. byorhinis strains and it suggests that considerable antigenic heterogeneity may exist amongst strains of this species. Although field strain 1 produced a unique antigenic profile against anti$M$. byorbinis strain GDL serum it was indistinguishable from the other three $M$. byorbinis strains (with the exception of a high molecular mass antigen at approximately $200 \mathrm{kDa}$ ) when reacted against anti- $M$. hyopneumoniae sera. Consequently, immunoblotting may be a useful tool for the identification of field strains of $M$. byorbinis and warrants further investigation, particularly in light of the studies performed by Gois $e t$ al. (1974), who described antigenic variation amongst seven strains of $M$. hyorbinis using a variety of immunological techniques. All four M. hyorhinis strains displayed marked antigenic size heterogeneity, producing a distinctive immunoreactive 'ladder' pattern on immunoblots. This antigen pattern has been reported for several species of mycoplasma including $M$. byorhinis and is most likely indicative of size variation of immunoreactive membrane associated lipoproteins described by Rosengarten \& Wise (1990).

M. hyopneumoniae strain C1735/2 is clearly unusual, displaying a unique protein profile, and is poorly immunoreactive against hyperimmune sera raised to either high or low passage strains of $M$. byopneumoniae. $\mathrm{C} 1735 / 2$ was recovered from lung samples submitted by a piggery (previously free of PEP) in south-east Queensland, Australia, when a severe outbreak of PEP occurred soon after the importation of boar from Canada. Two mycoplasma isolates (C1735/5, C1735/2) were recovered from infected lung and the gel-diffusion test identified C1735/5 as M. hyorbinis whilst C1735/2 failed to give any reaction with anti-M. hyorbinis or anti-M. byopneumoniae antisera. Strain $\mathrm{C} 1735 / 2$ was later identified as $M$. byopneumoniae by the growth inhibition test and its species identity confirmed by PCR using $M$. hyopneumoniae specific primers (this study). Avakian et al. (1991) described strains of Mycoplasma gallisepticum that were poorly immunoreactive to antiserum raised against another $M$. gallisepticum strain. 
These obervations suggest that some mycoplasma strains produce a structurally modified repertoire of antigens not recognized by some diagnostic antisera. Although hyperimmune sera failed to react strongly with $\mathrm{C} 1735 / 2$ antigens, rabbit anti-43 $\mathrm{kDa}$ antisera identified the $43 \mathrm{kDa}$ antigen in a whole cell lysate of $\mathrm{C} 1735 / 2$, confirming the identity of this strain as $M$. byopneumoniae. The poor immunoreactivity of strain C1735/2 highlights the limitations of gross serologically based typing methodologies and the requirement for more than one diagnostic antiserum to confirm species identity.

Whilst anti-M. hyopneumoniae J strain sera detected strongly immunoreactive $M$. byopneumoniae antigens of molecular mass between 52 and $62 \mathrm{kDa}$ in PPP experiments, these antigens are poorly reactive on immunoblots probed with the same antiserum. For both PPP and immunoblotting studies, antigens are denatured in reducing mixture (Laemmli, 1970) and separated by SDS-PAGE using the same buffer composition. Whilst proteins may partially renature during Western transfer (Bolske et al., 1987) the conformational state of mycoplasmal antigens dissolved in SDS-PAGE buffer, diluted in carbonate buffer and coated onto 96-well microtitre plates may be different compared with the same antigens used in immunoblotting studies. These differences may alter the repertoire of linear and conformational epitopes amongst different proteins, affecting the ability of some antibodies to bind to some antigens. Bolske $e t$ al. (1987) described different specificities of anti-M. byopneumoniae serum for antigens which had undergone different preparation procedures likely to affect their degree of renaturation. Finally, antigens identified as cross-reactive species in immunoblotting studies are often of a different molecular mass to those described in PPP studies, which may also be a reflection of this phenomenon.

Recently, we described M. byopneumoniae antigens of molecular masses of 76, 78, 80-82, 94, 106 and $114 \mathrm{kDa}$ which afforded a significant reduction in lung pathology when administered as parenteral vaccines to pigs (Djordjevic et al., 1996). Ultimately, the control of mycoplasmal pneumonia will depend upon the development of reliable diagnostic reagents and a thorough understanding of the mechanism(s) of pathogenesis. Recently, we have cloned and characterized a gene encoding the $94 \mathrm{kDa} M$. byopneumoniae-specific antigen described in this study. Hybridization studies have shown that the DNA encoding this antigen is present in all seven strains of $M$. hyopneumoniae described here yet fails to hybridize to genomic DNA from strains of $M$. flocculare and $M$. byorhinis (data not shown). More importantly, the deduced amino acid sequence reveals the presence of tandemly arranged, proline-rich repeat motifs typical of bacterial and eukaryote binding proteins (Williamson, 1994). Clearly, further studies are required to characterize the $M$. byopneumoniae-specific antigens of $36,43,76,94$, and $114 \mathrm{kDa}$ described here to evaluate their diagnostic and prophylactic potential as a means of controlling mycoplasmal pneumonia.

\section{ACKNOWLEDGEMENTS}

We are grateful for the supply of $M$. hyopneumoniae strain 232 from $\mathrm{Dr} T$. Young, French field isolate YZ from Dr M. Kobisch and strains C1735/2, Sue, Beaufort, OMZ407 from Dr A. Pointon, Australian Mycoplasma Reference Collection. A.S. is a recipient of an Australian Postgraduate Research Award. This work was partly funded by the Pig Research $\&$ Development Corporation (DAN 47P). We gratefully acknowledge the technical assistance of L. Romalis, $M$. Saunders and V. Taylor in photography and mycoplasmal culture.

\section{REFERENCES}

Andersen, H., Birkelund, S., Christiansen, G. \& Freundt, E. A. (1987). Electrophoretic analysis of proteins from Mycoplasma hominis strains detected by SDS-PAGE, 2-D gel electrophoresis and immunoblotting. J Gen Microbiol 133, 181-191.

Artiushin, S. \& Minion, F. C. (1996). Arbitrarily primed PCR analysis of Mycoplasma byopneumoniae field isolates demonstrates genetic heterogeneity. Int J Syst Bacteriol 46, 324-328.

Avakian, A. P., Kleven, S. H. \& Ley, D. H. (1991). Comparison of Mycoplasma gallisepticum strains and identification of immunogenic integral membrane proteins with Triton X-114 by immunoblotting. Vet Microbiol 29, 319-328.

Bereiter, M., Young, T. F., Joo, H. S. \& Ross, R. F. (1990). Evaluation of the ELISA and comparison to the complement fixation test and radial immunodiffusion enzyme assay for detection of antibodies against Mycoplasma byopneumoniae in swine serum. Vet Microbiol 25, 177-192.

Bolske, G., Strandberg, M. L., Bergstrom, K. \& Johansson, K. E. (1987). Species-specific antigens of Mycoplasma hyopneumoniae and cross reactions with other porcine Mycoplasmas. Curr Microbiol 15, 233-239.

Chan, H. W. \& Ross, R. F. (1984). Restriction endonuclease analyses of two porcine Mycoplasma deoxyribonucleic acids: sequence-specific methylation in the Mycoplasma byopneumoniae genome. Int J Syst Bacteriol 34, 16-20.

Christiansen, G. \& Andersen, H. (1988). Heterogeneity among Mycoplasma hominis strains as detected by probes containing parts of ribosomal ribonucleic acid genes. Int J Syst Bacteriol 38, 108-115.

Clyde, W. A. (1964). Mycoplasma species identification based upon growth inhibition by specific antisera. J Immunol 92, 958-965.

DeBey, M. C. \& Ross, R. F. (1994). Ciliostasis and loss of cilia induced by Mycoplasma hyopneumoniae in porcine tracheal organ cultures. Infect Immun 62, 5312-5318.

Djordjevic, S. P., Eamens, G. J., Romalis, L. F. \& Saunders, M. M. (1994). An improved enzyme linked immunosorbent assay (ELISA) for the detection of porcine serum antibodies against Mycoplasma hyopneumoniae. Vet Microbiol 39, 261-274.

Djordjevic, S. P., Eamens, G. J., Scarman, A. L. \& Chin, J. (1996). Characterisation of Mycoplasma byopneumoniae membrane proteins and identification of discrete antigens which significantly reduce lung pathology in vaccinated pigs challenged with a virulent strain of $M$. hyopneumoniae. IOM Lett 4, 282-283.

Freeman, M. J., Armstrong, C. H., Sands-Freeman, L. L. \& LopezOsuna, M. (1984). Serological cross-reactivity of porcine reference antisera to Mycoplasma byopneumoniae, M. flocculare, $M$. byorbinis and $M$. byosynoviae indicated by the enzyme-linked immunosorbent assay, complement fixation and indirect hemagglutination tests. Can J Comp Med 48, 202-207. 
Frey, J., Haldimann, A. \& Nicolet, J. (1992). Chromosomal heterogeneity of various Mycoplasma byopneumoniae field strains. Int J Syst Bacteriol 42, 275-280.

Friis, N. F. (1971). Mycoplasmas cultivated from the respiratory tract of Danish pigs. Acta Vet Scand 12, 69-79.

Friis, N. F. \& Feenstra, A. A. (1994). Mycoplasma hyorhinis in the etiology of serositis among piglets. Acta Vet Scand 35, 93-98.

Gois, M., Kuksa, F., Franz, J. \& Taylor-Robinson, D. (1974). The antigenic differentiation of seven strains of Mycoplasma byorbinis by growth-inhibition, metabolic-inhibition, latex-agglutination and polyacrylamide-gel-electrophoresis tests. J Med Microbiol 7, 105-115.

Goodwin, R. F. W., Pomeroy, A. P. \& Whittlestone, P. (1965). Production of enzootic pneumoniae in pigs with a mycoplasma. Vet Rec 77, 1247-1249.

Harlow, E. \& Lane, D. (editors) (1988). Antibodies : a Laboratory Manual. Cold Spring Harbor, NY: Cold Spring Harbor Laboratory.

lonas, G., Norman, N. G., Clarke, J. K. \& Marshall, R. B. (1991). A study of the heterogeneity of isolates of Mycoplasma ovipneumoniae from sheep in New Zealand. Vet Microbiol 29, 339-347.

Kazama, S., Yagihashi, T. \& Seto, Y. (1989). Preparation of Mycoplasma byopneumoniae antigen for the enzyme-linked immunosorbent assay. Can J Vet Res 53, 176-181.

Laemmli, U. K. (1970). Cleavage of structural proteins during the assembly of the head of bacteriophage T4. Nature 227, 680-685.

Mori, Y., Hamaoka, T., Sato, S. \& Takeuchi, S. (1988). Immunoblotting analysis of antibody response in swine experimentally inoculated with Mycoplasma hyopneumoniae. Vet Immunol Immunopathol 19, 239-250.

Poumarat, F., Solsona, M. \& Boldini, M. (1994). Genomic, protein and antigenic variability of Mycoplasma bovis. Vet Microbiol 40, 305-321.

Razin, S., Tully, J. G., Rose, L. \& Barile, M. F. (1983). DNA cleavage patterns as indicators of genetic heterogeneity among strains of Acholeplasma and Mycoplasma species. J Gen Microbiol 129, 1935-1944.

Ro, L. H. \& Ross, R. F. (1983). Comparison of Mycoplasma byopneumoniae strains by serologic methods. Am J Vet Res 44, 2087-2094.

Roberts, D. H. \& Little, T. W. A. (1970). Serologic studies in pigs with Mycoplasma byopneumoniae. J Comp Path 80, 211-220.

Rose, D. L., Tully, J. G. \& Wittler, R. G. (1979). Taxonomy of some swine mycoplasmas: Mycoplasma suipneumoniae Goodwin et al., 1965, a later, objective synonym of Mycoplasma byopneumoniae Mare and Switzer 1965, and the status of Mycoplasma flocculare Meyling and Friis 1972. Int J Syst Bacteriol 29, 83-91.

Rosengarten, R. \& Wise, K. S. (1990). Phenotype switching in mycoplasmas: phase variation of diverse surface lipoproteins. Science 247, 315-318.

Ross, R. F. (1992). Mycoplasmal diseases. In Diseases of Swine, pp. 537-551. Edited by A. D. Leman, B. Straw, W. T. Mengeling, S. D'Allaire \& D. J. Taylor. Ames, IA: lowa State University Press.

Ross, R. F. \& Young, T. F. (1993). The nature and detection of mycoplasmal immunogens. Vet Microbiol 37, 369-380.
Sheldrake, R. F. \& Romalis, L. F. (1992). Evaluation of an enzymelinked immunosorbent assay for the detection of Mycoplasma byopneumoniae antibody in porcine serum. Aust Vet $J$ 69, 255-258.

Städtlander, C. T. K.-H., Zuhua, C., Watson, H. L. \& Cassell, G. H. (1991). Protein and antigenic heterogeneity among strains of Mycoplasma fermentans. Infect Immun 59, 3319-3322.

Städtlander, C. T. K.-H. \& Watson, H. L. (1992). Protein and antigen variability amonst strains of Mycoplasma arthriditis. Zentralbl Bakteriol 276, 407-417.

Stemke, G. W., McIntyre, D. J., Roy, K. L., Stemler, M. E. \& Robertson, J. A. (1985). Guanine-plus-cytosine compositions of and deoxyribonucleic acid hybridisation comparisons between Mycoplasma byopneumoniae and Mycoplasma flocculare. Int J Syst Bacteriol 35, 527-529.

Stemke, G. W., Laiget, F., Grau, O. \& Bove, J. M. (1992). Phylogenetic relationships of three porcine mycoplasmas, Mycoplasma hyopneumoniae, Mycoplasma flocculare and Mycoplasma byorhinis and complete $16 \mathrm{~S}$ rRNA sequence of $M$. flocculare. Int J Syst Bacteriol 42, 220-225.

Stemke, G. W., Phan, R., Young, T. F. \& Ross, R. F. (1994). Differentiation of Mycoplasma hyopneumoniae, M. flocculare, and $M$. byorhinis on the basis of amplification of a $16 \mathrm{~S}$ rRNA gene sequence. Am J Vet Res 55, 81-84.

Strasser, M., Abiven, P., Kobisch, M. \& Nicolet, J. (1992). Immunological and pathological reactions in piglets experimentally infected with Mycoplasma byopneumoniae and/or Mycoplasma flocculare. Vet Immunol Immunopathol 31, 141-153.

Tajima, M. \& Yagihashi, T. (1982). Interaction of Mycoplasma byopneumoniae with the porcine respiratory epithelium as observed by electron microscopy. Infect Immun 37, 1162-1169.

Taylor-Robinson, D., Somerson, N. L., Turner, H. C. \& Chanock, R. M. (1963). Serological relationships among human mycoplasmas as shown by complement fixation and gel diffusion. J Bacteriol 85, 1261-1273.

Thirkell, D., Spooner, R. K., Jones, G. E., Russell, W. C. \& Voice, M. W. (1991). Cross-reacting antigens between Mycoplasma ovipneumoniae and other species of mycoplasma of animal origin, shown by ELISA and immunoblotting with reference antisera. Vet Microbiol 26, 249-261.

Towbin, J., Staehelin, T. \& Gordon, J. (1979). Electrophoretic transfer of proteins from polyacrylamide gels to nitrocellulose sheets: procedure and some applications. Proc Natl Acad Sci USA 76, 4350-4354.

Williamson, M. P. (1994). The structure and function of prolinerich regions in proteins. Biochem J 297, 249-260.

Young, T. F. \& Ross, R. F. (1987). Assessment of antibody response of swine infected with Mycoplasma hyopneumoniae by immunoblotting. Am J Vet Res 48, 651-656.

Zhang, Q., Young, T. F. \& Ross, R. F. (1995). Identification and characterisation of a Mycoplasma byopneumoniae adhesin. Infect Immun 63, 1013-1019.

Zielinski, G. C. \& Ross, R. F. (1990). Effect of growth in cell cultures and strain on virulence of Mycoplasma byopneumoniae for swine. Am J Vet Res 51, 344-348.

Received 10 June 1996; revised 26 September 1996; accepted 2 October 1996. 